\title{
Membrane-based torque magnetometer: Enhanced sensitivity by optical readout of the membrane displacement
}

M. Blankenhorn, E. Heintze, M. Slota, J. van Slageren, B. A. Moores, C. L. Degen, L. Bogani, and M. Dressel

Citation: Review of Scientific Instruments 88, 094707 (2017); doi: 10.1063/1.5004045

View online: https://doi.org/10.1063/1.5004045

View Table of Contents: http://aip.scitation.org/toc/rsi/88/9

Published by the American Institute of Physics

\section{Articles you may be interested in}

The metal-insulator transition in the organic conductor $\beta$ "-(BEDT-TTF) ${ }_{2} \mathrm{Hg}(\mathrm{SCN})_{2} \mathrm{Cl}$

The Journal of Chemical Physics 147, 064503 (2017); 10.1063/1.4997198

Torque magnetometry in pulsed magnetic fields with use of a commercial microcantilever Review of Scientific Instruments 73, 3022 (2002); 10.1063/1.1491999

Nuclear quantum-assisted magnetometer

Review of Scientific Instruments 88, 013702 (2017); 10.1063/1.4973449

Simple torque magnetometer for anisotropy measurements in magnetic thin-film media

Review of Scientific Instruments 59, 2265 (1988); 10.1063/1.1139945

Torsional frequency mixing and sensing in optomechanical resonators

Applied Physics Letters 111, 111102 (2017); 10.1063/1.4986811

Torque magnetometry of perpendicular anisotropy exchange-spring heterostructures Journal of Applied Physics 120, 013903 (2016); 10.1063/1.4955041

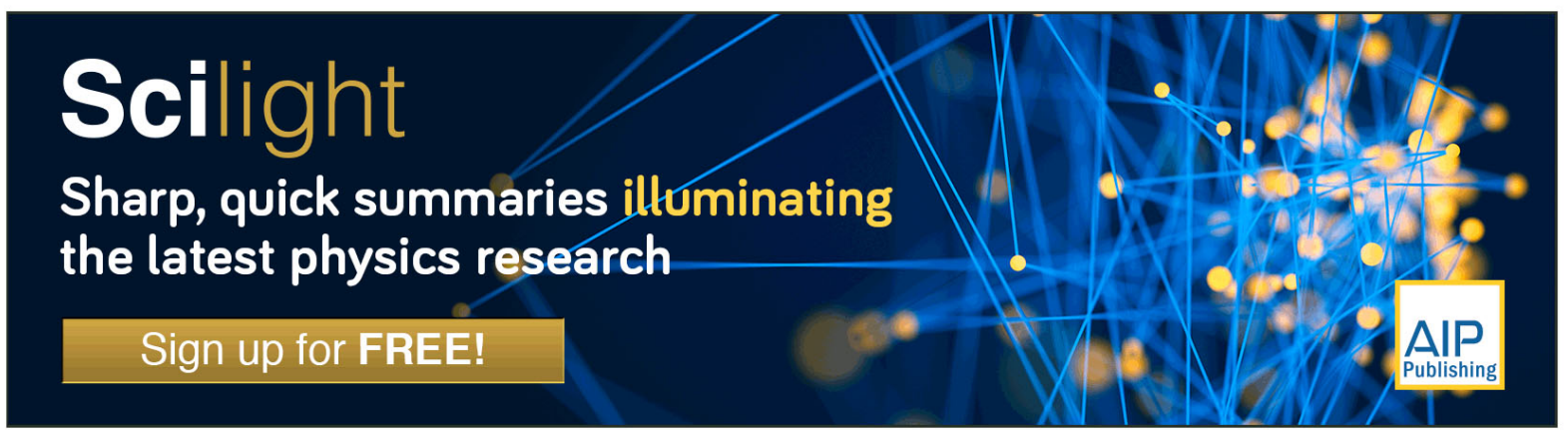




\title{
Membrane-based torque magnetometer: Enhanced sensitivity by optical readout of the membrane displacement
}

\author{
M. Blankenhorn, ${ }^{1, a)}$ E. Heintze, ${ }^{1}$ M. Slota,,${ }^{1, b)}$ J. van Slageren, ${ }^{2}$ B. A. Moores,,${ }^{3, c)}$ \\ C. L. Degen, ${ }^{3}$ L. Bogani, ${ }^{1, b)}$ and M. Dressel ${ }^{1}$ \\ ${ }^{1}$ 1. Physikalisches Institut, Universität Stuttgart, 70550 Stuttgart, Germany \\ ${ }^{2}$ Institut für Physikalische Chemie, Universität Stuttgart, 70550 Stuttgart, Germany \\ ${ }^{3}$ Department of Physics, ETH Zürich, 8093 Zürich, Switzerland
}

(Received 20 May 2017; accepted 8 September 2017; published online 27 September 2017)

\begin{abstract}
The design and realization of a torque magnetometer is reported that reads the deflection of a membrane by optical interferometry. The compact instrument allows for low-temperature measurements of tiny crystals less than a microgram with a significant improvement in sensitivity, signal-to-noise ratio as well as data acquisition time compared with conventional magnetometry and offers an enormous potential for further improvements and future applications in different fields. Magnetic measurements on single-molecule magnets demonstrate the applicability of the membrane-based torque magnetometer. Published by AIP Publishing. https://doi.org/10.1063/1.5004045
\end{abstract}

\section{INTRODUCTION}

A wide range of experimental techniques are available to investigate magnetic properties of materials, even many commercial instruments provide an unprecedented sensitivity. Most commonly used apparatuses are superconducting quantum interference devices (SQUID), Foner's vibrating sample magnetometers, Hall probes, and torque magnetometers. ${ }^{1-3}$ The last of these offer high sensitivity and are mostly employed for the study of single crystals or thin films. ${ }^{4-7}$ Torque magnetometry is an important tool in today's condensed matter physics, in particular, in the field of nanomagnetic materials, superconductors, topological insulators, and heavy fermion systems.

Two types of torque magnetometers are employed. The first uses a cantilever beam as torque sensor, while the second type uses a thin wire attached to the sample, and the torsion is recorded. Several detection schemes have been developed, e.g., capacitive, ${ }^{7-10}$ optical, ${ }^{11-13}$ or piezoresistive methods. ${ }^{14-16}$ During the past years, torque sensors were miniaturized in micro/nano-lever systems pushing the sensitivity down to $10^{-14} \mathrm{Am}^{2}$. However, the systems became extremely complex, and these ultra-precise methods impose severe restrictions on sample size and accessibility.

Here, we present a novel type of torque magnetometer, which utilizes an ultra-thin silicon nitride $(\mathrm{SiN})$ membrane as a torque sensor combined with optical detection of displacements, offering a sensitivity down to $2 \times 10^{-13} \mathrm{Am}^{2} / \sqrt{\mathrm{Hz}}$ (see Appendix C). The compact and simple design allows for optical access, and the magnetometer can be operated down to milli-Kelvin temperatures but still realized at low costs. The detection via a fiber-optical Fizeau interferometer enables fast

\footnotetext{
a)Present address: Institut für Funktionelle Materie und Quantentechnologie, Universität Stuttgart, 70550 Stuttgart, Germany.

b)Present address: Department of Materials, University of Oxford, Oxford OX1 3PH, United Kingdom.

c)Present address: JILA, National Institute of Standards and Technology and the University of Colorado, Boulder, Colorado 80309, USA.
}

acquisition to measure dynamics on short time scales compared with common averaging techniques. In the following, we discuss the experimental implementation of the membrane as a torque sensor. We demonstrate the capability and sensitivity of the membrane torque magnetometer (MTM) by measurements on the well-known single-molecule magnet $\mathrm{Mn}_{12}$ ac.

\section{BASIC PRINCIPLE}

While common devices such as SQUIDs and Hall probes sense the magnetic moment indirectly via the magnetic stray field, torque magnetometers directly detect the magnetic moment of the sample. Here, the measured quantity is the magnetic torque,

$$
\tau=\boldsymbol{m} \times \boldsymbol{B},
$$

caused by the fact that the sample's net magnetic moment $\boldsymbol{m}$ is not collinear with the applied magnetic field $\boldsymbol{B}$, which is assumed uniform at this point. This non-collinearity usually results from a magnetic anisotropy that pins the magnetic moment to a specific direction in the particle. In the weak-field limit, the magnetic susceptibility does not depend on the magnetic field; thus, the vector product can be written as ${ }^{17} \tau=\frac{1}{2} B^{2}\left(\chi_{i}-\chi_{j}\right)$ sin $2 \theta$, where $\chi_{i}$ and $\chi_{j}$ denote the magnetic susceptibilities along the principle magnetic axes $i$ and $\boldsymbol{j}$, respectively. Further, $\theta$ is the angle between the magnetic field $\boldsymbol{B}$ and the $i$-axis, with $\boldsymbol{B}$ lying in the $(i j)$-plane. As the magnetic torque relies on a preferred orientation of the magnetic moment, it is not affected by an isotropic background that often poses problems for other magnetometry techniques.

Torque setups are commonly based on cantilever beams or torsion wires. Here, we follow a novel approach utilizing a thin $\mathrm{SiN}$ membrane as a sensitive torque meter. ${ }^{12}$ The sample is fixed to the membrane; therefore, a torque applied to the sample causes a deflection in the diaphragm. While the conventional cantilever arm is fixed at one end and can be treated mathematically by a one-dimensional differential equation, the 
membrane is a stretched foil clamped on all sides. Applying a force at a certain position constitutes a far more complex situation leading to a rather intriguing response in three dimensions. A good starting point is the analytical solution for the deflection of a circular diaphragm used as pressure sensors presented by Eaton et al. ${ }^{18}$ details are given in Appendix A. It reveals that the bending is inversely proportional to the builtin strain of the membrane $\varepsilon_{i}$ as well as its thickness $h$. While the behavior is independent of the membrane surface area, the deflection is linear with applied force up to $5 \mu \mathrm{m}$.

For the present purpose, numerical simulations and visualizations appear more instructive; hence, we conducted a finite-element analysis with regard to sample position and geometry. The details of the model are given in Appendix A. We should note, however, that this model describes only very small displacements correctly and does not consider any tensile stress. Figure 1 illustrates the response of the membrane, when a magnetic sample is attached to it. If the specimen is located in the center [Fig. 1(a)], in the presence of a magnetic field opposite forces act on both ends of the crystal leading to an indention of the membrane on one side and a raise on the other. Only if the crystal under study is positioned off center close to the edge of the diaphragm, it pulls or pushes the membrane forming a single bump in the center, as illustrated in Fig. 1(b).

In order to measure the tiny displacements $\Delta d$ of the membrane, we employed a Fizeau-type interferometer, which has already been implemented successfully by Rugar and others in the context of atomic-force microscopy. ${ }^{19-21}$ Monochromatic light is guided to the center of the membrane via a singlemode optical fiber: a small fraction of the light is reflected right at the cleaved end of the quartz fiber (refractive index
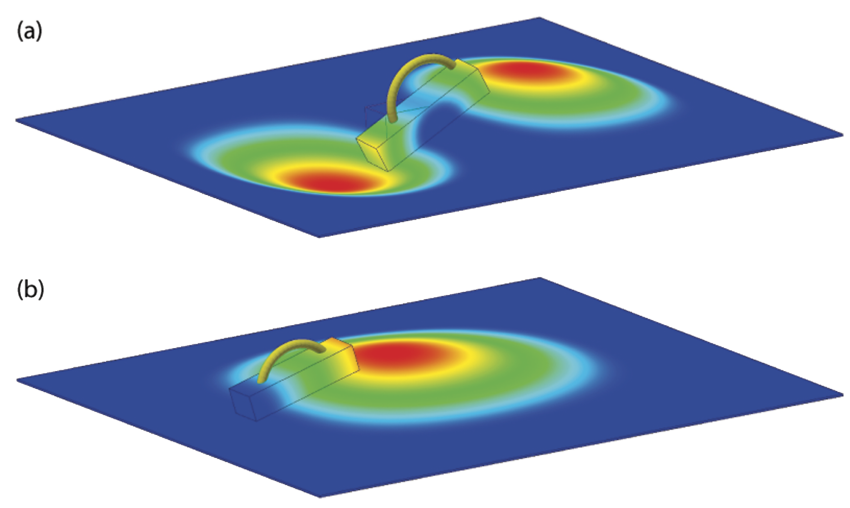

FIG. 1. Finite element analysis of the deflection when a sample is placed onto the membrane and experiences a certain torque; in our case, the area shown corresponds to approximately $1 \mathrm{~mm} \times 1 \mathrm{~mm}$. The simulation is obtained by the Autodesk Inventor 2016 stress analysis tool using the material properties of the SiN foil: density $\rho=3.18 \mathrm{~g} / \mathrm{cm}^{3}$, Young's modulus $E=427.18 \mathrm{GPa}$, and Poisson's ratio $v=2.3$. The software does not consider nonlinear effects introduced by stress in the material and thus does not allow quantitative estimates of the response. Beside this restriction the software enables us to apply a torque on a certain crystal geometry and, therefore, to qualitatively simulate the response with respect to the sample position. (a) When the crystal is placed in the center of the membrane with a magnetic field applied perpendicular, it experiences a torque-indicated by the golden ring-that pulls up one side, while it pushes down the opposite side. (b) If the crystal is located on one side of the membrane, the force lifts only the central part. $n=1.444$ at $\lambda=1550 \mathrm{~nm}$ ) and comprises one of the two interfering beams. About $96 \%$ of the light exits the fiber and is reflected off the membrane surface. A part of the reflected light reenters the fiber. This optical detection scheme allows for a precise and continuous measurement of sub-nanometer deflections. By using both a laser diode as light source and an all-fiber construction, the sensor features a small size while still maintaining full mechanical robustness. The system can be operated in an ambient environment but also in vacuum; and it can be cooled down to low temperatures. Since the light propagates through fibers except for the small gap between the tip and the membrane, the sensor is insusceptible to air turbulences, in particular, to acoustic noise.

The two interfering waves are guided back into the optical fiber and detected by a photoreceiver. Its output voltage $V$ is given by $V=V_{m}-V_{0} \cos \{4 \pi(d+\Delta d) / \lambda\}$ with fibermembrane spacing $d$ and laser wavelength $\lambda$. The parameters $V_{m}=\frac{1}{2}\left(V_{\max }+V_{\min }\right)$ and $V_{0}=\frac{1}{2}\left(V_{\max }-V_{\min }\right)$ correspond to the mean value and amplitude, respectively. The sensor is most sensitive where the slope of the interference signal is the steepest, i.e., where the phases of the two reflected components are in quadrature: $d=\lambda / 8,3 \lambda / 8,5 \lambda / 8, \ldots$. At these points of operation, a small variation $\Delta d$ transforms into a significant change in output voltage yielding a linear relation between voltage and distance,

$$
\Delta V=V_{0} \sin \left\{\frac{4 \pi \Delta d}{\lambda}\right\} \approx V_{0} 4 \pi \frac{\Delta d}{\lambda} .
$$

The signal strength crucially depends on the interference amplitude $V_{0}$. At a wavelength of $1.5 \mu \mathrm{m}$, the reflectivity of the $\mathrm{SiN}$ is $38 \%$, and the optimal distance of fiber tip to membrane is $d_{\text {opt }} \approx 105 \mu \mathrm{m}$, leading to a spot diameter of $14.5 \mu \mathrm{m}$.

\section{EXPERIMENTAL SETUP}

As a torque sensor, we use low-stress SiN membranes, which are frequently deployed as vacuum windows in x-ray spectroscopy or sample holders for transmission electron microscopy. In recent years they also became popular in other fields, e.g., to study quantum effects in opto-mechanical systems, as nano-torsional resonators or for membrane-based nano-calorimeters. ${ }^{11,12,22-24}$ The membranes are readily available in different thicknesses ranging from 5 to $1000 \mathrm{~nm}$. We chose the model NX5150C by Norcada, Inc. ${ }^{25}$ featuring a $1.5 \times 1.5 \mathrm{~mm} \mathrm{SiN}$ square membrane with a thickness of 100 $\mathrm{nm}$, embedded in a $5 \times 5 \mathrm{~mm}$ silicon frame, as illustrated in Fig. 2(a). Since a variety of membrane models with different geometries are available, we can select the one that fits to the sample requirements best, for instance, a particular sample geometry or signal intensity. All diaphragms come with a tensile stress of approximately $(250 \pm 50) \mathrm{MPa}$ causing a built-in strain $\mu_{i}=\sigma / E=250 \mathrm{MPa} / 427 \mathrm{GPa}=0.59 \times 10^{-3}$, where $E$ is Young's modulus.

The optical interferometer consists of the laser diode package model QDFBLD-1550-5 supplied by QPhotonics LLC ${ }^{27}$ and operating at a wavelength of $\lambda=1550 \mathrm{~nm}$, a 99:1 fiber coupler 10202A-99-APC and a single mode fiber CCC1310-J9 purchased from Thorlabs, ${ }^{28}$ and a low-noise variable gain photoreceiver OE-200-IN2-FC obtained from Femto $\mathrm{GmbH},{ }^{29}$ 

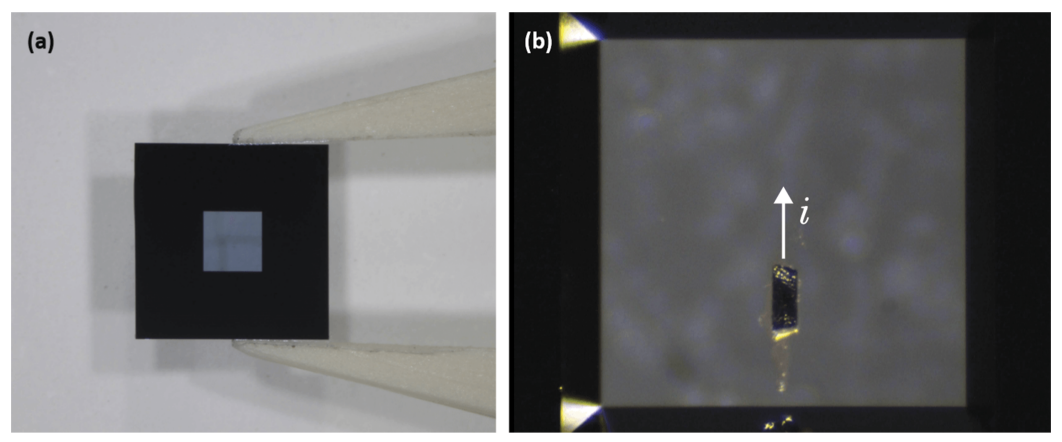

FIG. 2. (a) Optical photograph of the SiN membrane in a silicon frame. The membrane $(1.5 \mathrm{~mm} \times 1.5 \mathrm{~mm})$ has a thickness of $100 \mathrm{~nm}$ and is supported by a $\mathrm{Si}$ frame $5 \mathrm{~mm} \times 5 \mathrm{~mm}$ of $0.2 \mathrm{~mm}$ thickness. (b) Photograph of a $\mathrm{Mn}_{12}$ ac single crystal on the SiN diaphragm $\left(1.5 \times 1.5 \mathrm{~mm}^{2}\right)$. The crystal has a rod-like shape pointing along the crystallographic $c$-axis, representing the magnetic easy axis $i^{26}$

consisting of a InGaAs PIN photodiode delivering a maximum output of $10 \mathrm{~V}$ (mostly operated with a conversion gain of $1 \times 10^{7} \mathrm{~V} / \mathrm{W}$ where the bandwidth is $50 \mathrm{kHz}$ ). The laser diode package includes a thermoelectric cooler and thermistor

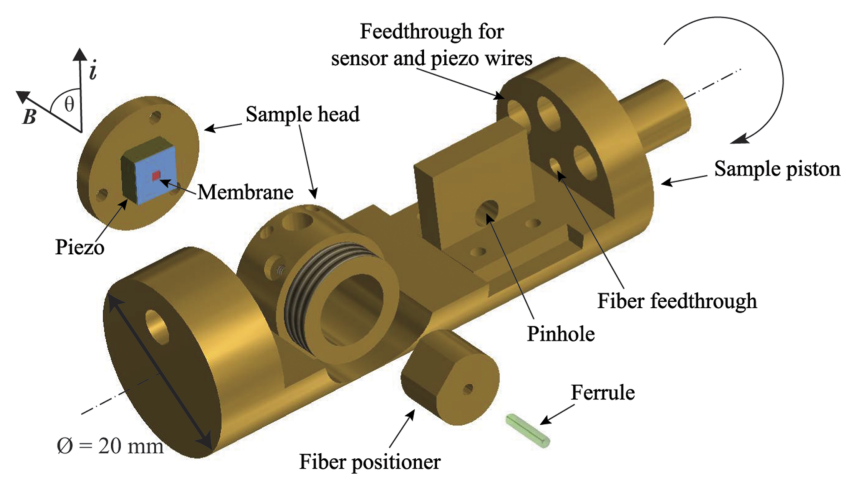

FIG. 3. Illustration of the piston and sample head designed to fit into the sample compartment of a helium flow cryostat. The sample piston features a central feedthrough for the fiber, wires to contact the piezo element, and a temperature sensor. The opening at the bottom ensures a stable gas flow and reduces turbulences. The sample head enables alignment of the fiber with respect to the membrane. The tubular design allows rotation around the central axis to vary the angle between the magnetic field and membrane plane, with the easy axis $i$ lying in the membrane plane. The entire insert can be translated up and down along the axis in order to place the sample in the central position of the magnetic field. Furthermore, the pinhole allows for optical transmission measurements. This way the frequency dependent output power of the microwave source can be monitored when torque-detected electron spin resonance spectroscopy is performed. Additionally, it simplifies the alignment of the sample probe with respect to the microwave beam. to stabilize and control the operational temperature. The laserdiode pump current is supplied by the laser diode controller LDC201CU by Thorlabs. A Rohde \& Schwarz SMG signal generator modulates the pump current at a frequency of $17 \mathrm{MHz}$ in order to reduce the coherence length of the laser. Decreasing the laser coherence suppresses other modes in the fiber, which makes us only sensitive to the small mode formed between our fiber and sample. Thanks to the low bend-loss specifications of the chosen fiber, a $5 \mathrm{~mm}$ bending radius attenuates the signal less than $0.1 \mathrm{~dB}$.

The torque magnetometer can be operated in conventional electromagnets, superconducting magnet systems, and dilution refrigerators. The scheme displayed in Fig. 3 was designed to fit into a magneto-optical cryostat Oxford Instruments Spectromag 4000 in order to conduct magnetization measurements down to low temperatures $(1.7-300 \mathrm{~K})$ and up to high magnetic fields $(9 \mathrm{~T})$. The setup provides an additional optical access required to study light-induced effects in magnetic materials. The main part is machined out of a single piece of brass, with a diameter $D=20 \mathrm{~mm}$ and length $L=55 \mathrm{~mm}$, to ensure stability and good thermal coupling. It can be rotated within the cryostat by a stepper motor to adjust the sample with respect to the horizontal magnetic field. The fiber is fed through the supporting tube (4 $\mathrm{mm}$ diameter) into the sample chamber that contains the sample head with the torque sensor. Vacuum tight sealing is achieved by Teflon (PTFE) tape and Apiezon M grease as shown in Fig. 4(b).

As seen in the drawing of Fig. 3, the design of the sample head enables us to align the fiber towards the center of the membrane torque sensor. The cleaved fiber is glued into a
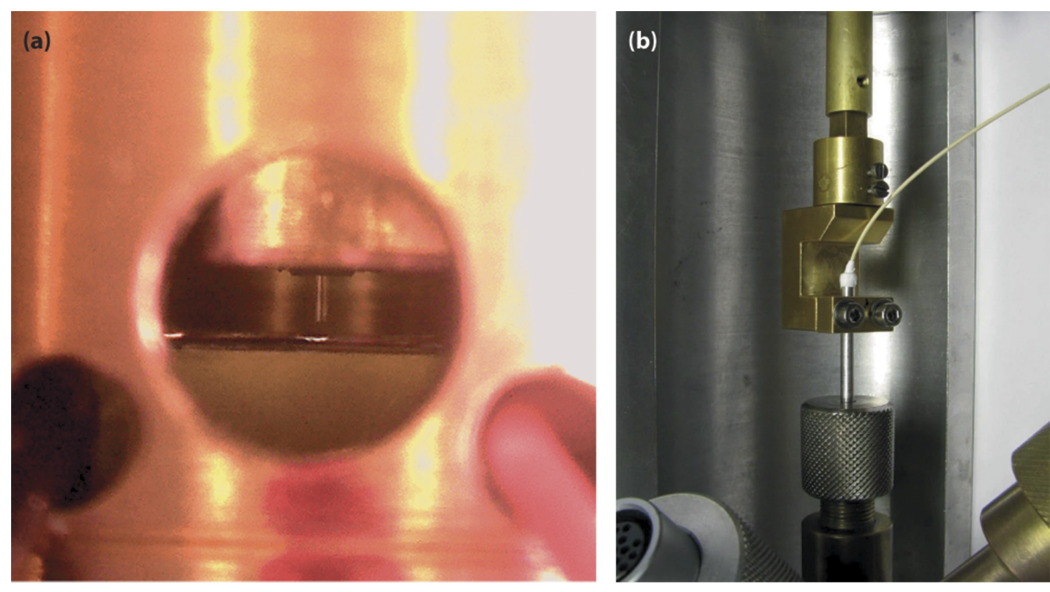

FIG. 4. (a) The spacing of fiber (top) to membrane (bottom) can be monitored through a hole in the sample head. Here, the distance is around $100 \mu \mathrm{m}$. (b) The photograph of the tube holder design illustrating how the fiber is fed into the piston tube. The fiber is sealed by a Teflon tape. In order to revolve the sample piston, the entire piece can be rotated without breaking the fiber. The stepper motor is connected via an adjustable adapter to enable translations along the rotational axis. 
ceramic ferrule for better handling. The membrane is placed on a piezoelectric chip (travel range: $2.8 \mu \mathrm{m}$ at room temperature, but only $300 \mathrm{~nm}$ at helium temperature, ${ }^{30}$ operated by a Keithley 2400 sourcemeter up to $75 \mathrm{~V}$ ) to adjust the fiber-membrane spacing to the operation point of the interferometer, where the phases of the two reflected light components are in quadrature [see Eq. (2)]. Once, this position is reached, the voltage applied to the piezoelectric chip is fixed. The piezo is permanently glued to the brass base plate. For easy exchange of the membrane, we fix it to the piezo element by grease. Additional silicon spacers between membrane and piezoelectric chip enable pressure compensation. Otherwise helium flow and pressure variations between the two sides of the membrane might cause substantial noise or even damage of the SiN membrane.

\section{EXPERIMENTAL PREPARATION}

As we have seen from our finite-element analysis, the correct position and alignment of the sample is essential for performing torque measurements. Due to the small thickness, the $\mathrm{SiN}$ membrane is very fragile, making the sample preparation challenging. The micrometer stage of a four-point probe station provides precise control in all three spatial directions together with a $50 \mu \mathrm{m}$ thin wire. This way we precisely deposit a small amount of low-temperature vacuum grease on the membrane. In a second step the sample under study is placed on the grease spot. While the crystal can still be aligned and slightly moved using the micrometer stage, the grease freezes at cryogenic temperatures and thus provides stable mounting of the sample onto the membrane.

The loaded membrane is then put on the piezoelectric chip, once again utilizing vacuum grease to steady it. To adjust the cleaved fiber to the membrane surface, it is glued into a ceramic ferrule, which snugly fits the hole drilled in the positioner (Fig. 3). Employing a micrometer stage the fiber can be gradually traversed in the direction of the membrane. The fiber-membrane spacing is monitored via a USB camera through a hole, as illustrated in Fig. 4(a), as well as by the interferometer signal itself. The optimum fiber-membrane spacing is achieved when the interference minimum takes the lowest possible value. Once the correct position is reached, the fiber positioner is locked by a set screw. Optionally, the fiber position can be checked by driving the piezoelectric chip. This also reveals the maximum and minimum interferometer signals under constructive and destructive interference conditions, which is necessary when analyzing the raw data. The fiber alignment procedure has to be followed once, as the sample can easily be exchanged by simply removing the bottom plate of the sample head and substituting the membrane by another one prepared externally.

\section{TEST MEASUREMENTS}

In order to test the novel membrane-based torque magnetometer, we have chosen the best studied singlemolecule magnet $\mathrm{Mn}_{12}$ ac as a sample, which stands for $\left[\mathrm{Mn}_{12} \mathrm{O}_{12}\left(\mathrm{CH}_{3} \mathrm{COO}\right)_{16}\left(\mathrm{H}_{2} \mathrm{O}\right)_{4}\right] \cdot 2 \mathrm{CH}_{3} \mathrm{COOH} \cdot 4 \mathrm{H}_{2} \mathrm{O}{ }^{31}$ The magnetic core consists of eight manganese(III) ions (spin $S=2$ ) and four manganese(IV) ions $(S=3 / 2)$, which are coupled antiferromagnetically resulting in a total spin ground state of $S=10$. In a magnetic field the energies of the ground state are described by the spin Hamiltonian $\mathcal{H}_{C F}=D S_{z}^{2}+B_{4}^{0} \boldsymbol{O}_{4}^{0}+$ $B_{4}^{4} \boldsymbol{O}_{4}^{4}+g \mu_{B} \boldsymbol{S B}$ with the axial zero field splitting parameter $D / k_{B}=0.66 \mathrm{~K}$, the fourth rank parameters $B_{4}^{0} / k_{B}=(3.2 \pm 0.2)$ $\times 10^{-5} \mathrm{~K}$ and $B_{4}^{4} / k_{B}=( \pm 6 \pm 1) \times 10^{5} \mathrm{~K}$ and the $g$-factor $g \approx 2 .{ }^{32}$ Due to the high energy barrier $D S^{2} / k_{B}=66 \mathrm{~K}$, the slow relaxation and quantum tunneling effects can be observed already at temperatures of about $3 \mathrm{~K} \cdot{ }^{17,26,32-35}$

Figure 2(b) shows a photograph of the investigated single crystal mounted onto the SiN membrane with the help of vacuum grease. During the cooling process of the system, the interferometer signal passes through several interference cycles, as the brass components, the vacuum grease and the used adhesives experience thermal contraction with decreasing temperature. Additionally, thermal contraction and degassing of the vacuum grease leads to sudden jumps in the signal. The temperature dependence of the signal slows down with decreasing temperature until it becomes basically temperature independent below $15 \mathrm{~K}$. Thereby the noise level of the

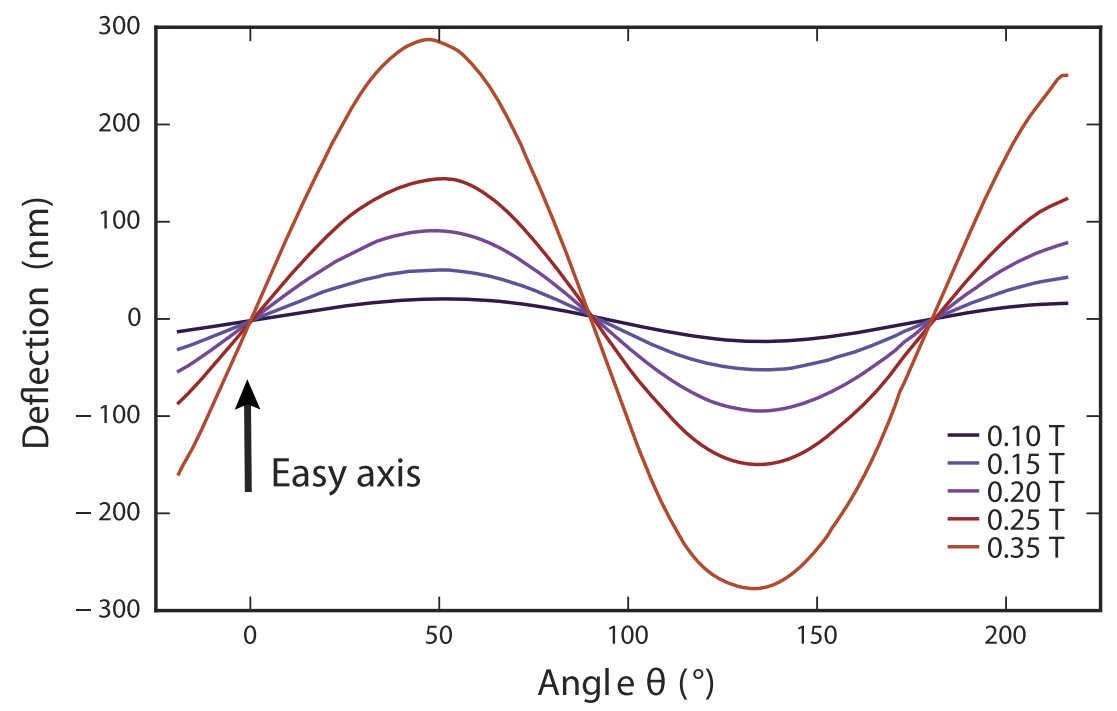

FIG. 5. Test measurement of a single crystal of the molecule magnet $\mathrm{Mn}_{12}$ ac. The data are taken at $T=9 \mathrm{~K}$ with different magnetic fields as indicated. Variation of the membrane deflection is shown as the angle between membrane and external magnetic field is varied. The easy axis is located at $0^{\circ}$. 
interferometer stays constant at $10 \mathrm{mV}$, which corresponds to a membrane deflection of $0.25 \mathrm{~nm}$. In the following we present angle and field dependent measurements on the $\mathrm{Mn}_{12}$ ac single crystal for different temperatures and magnetic field sweep rates.

\section{A. Angle-dependent measurements}

When the crystal is rotated in a constant magnetic field, the resulting torque varies with the angle between the

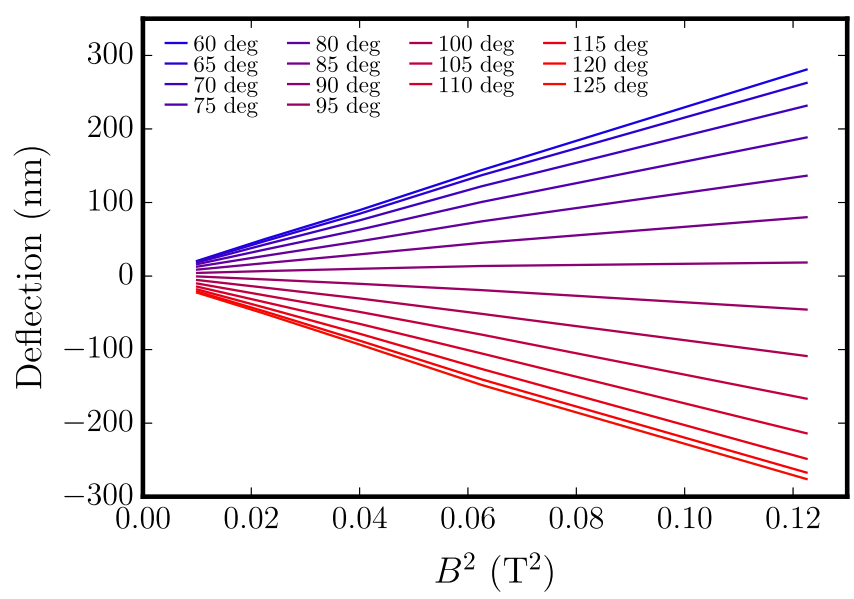

FIG. 6. Membrane deflection $\Delta d \propto \Delta V$ plotted as a function of the magnetic field squared for different angles of rotation recorded on a $\mathrm{Mn}_{12}$ ac single crystal at $T=1.8 \mathrm{~K}$. magnetization axis and the external field $\boldsymbol{B}$, according to Eq. (1). The interferometer signal is collected continuously while the sample head revolves in the field. In this way the acquisition time is reduced to about $90 \mathrm{~s}$ for a $180^{\circ}$ rotation with a resolution of $0.4^{\circ}$. With a reduced step rate at the motor, an accuracy of up to $0.05^{\circ}$ can be achieved. Using a python-based homemade software program, multiple angledependent measurements at different fields are conducted in an automatized manner. The actual membrane deflection $\Delta d$, which is proportional to the magnetic torque, is calculated from the interferometer signal using Eq. (2). Figure 5 displays the angular dependence for various magnetic fields ranging from $B=0.1 \mathrm{~T}$ to $0.35 \mathrm{~T}$, recorded at $T=9 \mathrm{~K}$. The curves exhibit a sinusoidal behavior with twofold periodicity as expected for a magnetically anisotropic easy-axis system. From the intersections of curves taken at different fields, we can determine the angle between the easy axis and the applied magnetic field. Figure 6 demonstrates the quadratic field dependence of the torque signal in the weak-field regime, which is obeyed for all angles measured.

\section{B. Field-dependent measurements}

When going to higher magnetic fields, $\mathrm{Mn}_{12}$ ac reveals its peculiar behavior due to quantum tunneling of the magnetization at low temperatures. ${ }^{31}$ Thus, we performed fielddependent magnetic torque measurements on $\mathrm{Mn}_{12}$ ac at a fixed angle of $4.3^{\circ}$. At different temperatures ranging from $3 \mathrm{~K}$ to $13 \mathrm{~K}$, the magnetic field was driven from $-1.9 \mathrm{~T}$ to $+1.9 \mathrm{~T}$

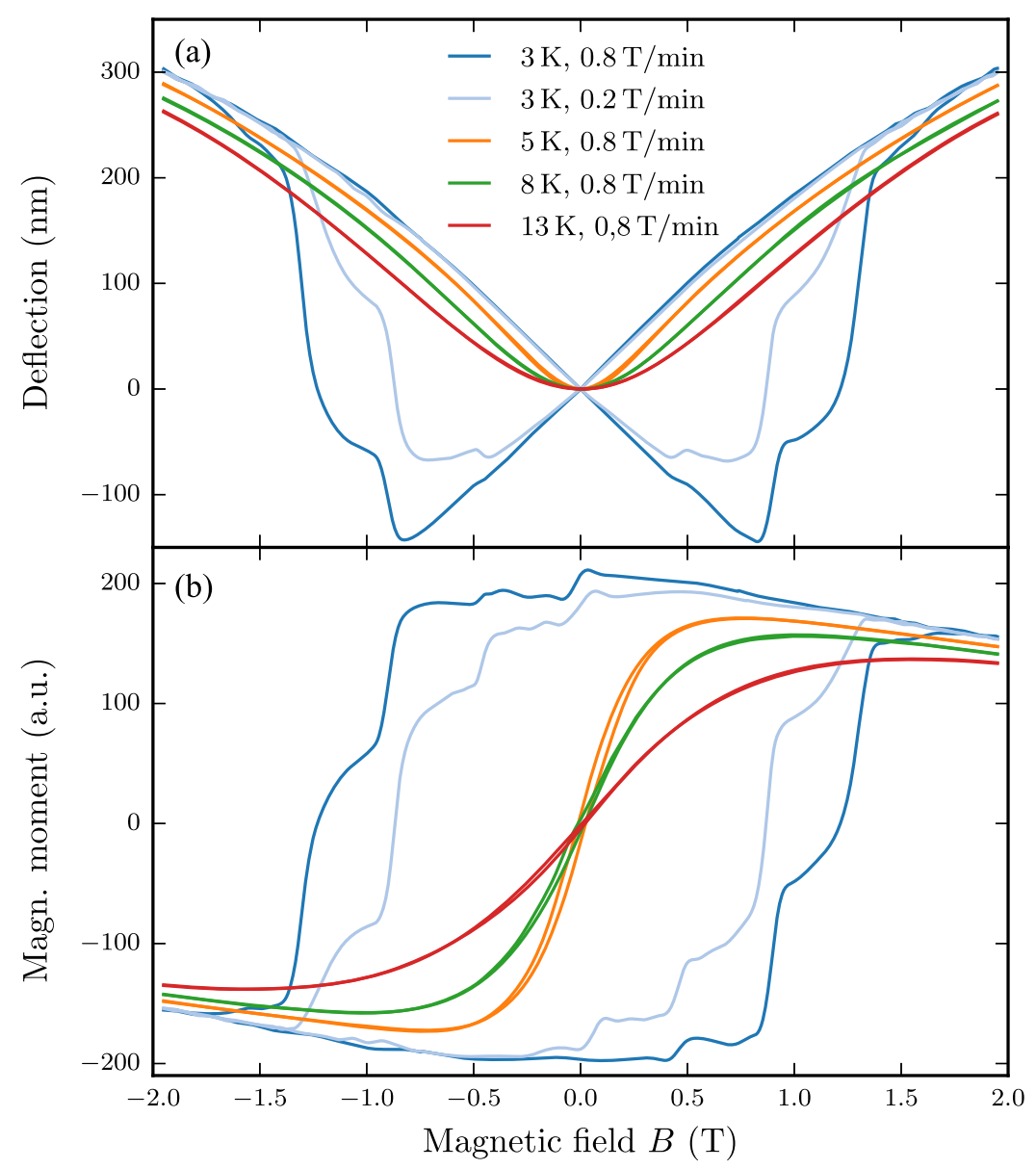

FIG. 7. (a) Magnetic-field dependence of the deflection measured on a $\mathrm{Mn}_{12}$ ac single crystal at different temperatures and sweep rates as indicated. (b) The magnetic moment of $\mathrm{Mn}_{12} \mathrm{ac}$ as a function of the magnetic field as obtained from membrane-based torque measurements. For $T \leq 3 \mathrm{~K}$ steps in the hysteresis curve become clearly visible due to quantum tunneling of magnetization in $\mathrm{Mn}_{12}$ ac single-molecule magnets. ${ }^{36}$ 
and back again with a rate of $0.8 \mathrm{~T} / \mathrm{min}$. To determine the dependence on the sweep rate, an additional measurement was conducted at $T=3 \mathrm{~K}$ with a rate of $0.2 \mathrm{~T} / \mathrm{min}$. In Fig. 7(a) the deflection is plotted versus external magnetic field, where the data were already corrected for a remanent field of the magnet of approximately $B_{r}=6 \mathrm{mT}$.

At elevated temperatures no hysteresis is present, and the deflection follows the expected behavior. In the weak-field limit-below $0.5 \mathrm{~T}$ - the signal increases quadratically and then changes to a linear behavior as the magnetic moment of the sample crystal saturates. For magnetic fields above $1 \mathrm{~T}$, however, the deflection starts to converge approaching a saturation value determined by the magnetic anisotropy of the $\mathrm{Mn}_{12}$ ac crystal. ${ }^{33}$ For small magnetic fields $B<1 \mathrm{~T}$, where the torque signal is proportional to the magnetic field, the field dependent behavior of the magnetic moment can be determined by dividing the deflection $\Delta d$ by the magnetic field $B$ as presented in Fig. 7(b). The magnetic moment calculated in that manner decreases slowly towards zero as the magnetic field increases.

Around $T \approx 5 \mathrm{~K}$ a small hysteresis gap starts to open up and increases further as the temperature is lowered; below $3 \mathrm{~K}$ the hysteresis loop ranges from approximately $-1 \mathrm{~T}$ to $+1 \mathrm{~T} .{ }^{36}$ Most interesting, abrupt steps appear in the magnetization curve due to quantum tunneling of the magnetization. These steps in magnetization occur at magnetic field values,

$$
\begin{array}{ll}
B_{1}=0.030 \mathrm{~T}, & B_{3}=0.885 \mathrm{~T}, \\
B_{2}=0.465 \mathrm{~T}, & B_{4}=1.320 \mathrm{~T},
\end{array}
$$

in excellent agreement with previous results. ${ }^{37,38}$ Care is required when interpreting the data around zero magnetic field, as very small values are divided. Hence some points close to zero need to be disregarded. A great advantage of the setup is that the deflection is continuously measured and thus fast relaxation processes can be investigated much better than by standard SQUID magnetometry. In Appendix B we compare the data obtained by our novel membrane-based torque magnetometer with SQUID data obtained on $\mathrm{Mn}_{12}$ ac. Additional improvements in the sensitivity can be achieved by the implementation of lock-in and averaging techniques.

\section{CONCLUSION AND OUTLOOK}

Here, we present the design and realization of a novel torque magnetometer based on a SiN membrane combined with highly sensitive optical displacement detection. The systems operate at helium temperatures and in magnetic fields up to $8 \mathrm{~T}$ and higher. Using simulations of the membrane by a finite-element analysis and by an analytical approach, we determine the ideal sample position and reveal a linear torque deflection behavior up to a deflection of $5 \mu \mathrm{m}$. To test the system we compare the results with standard SQUID magnetometry. The signal-to-noise ratio is superior, and angledependent measurements become faster by a factor of 200 with no losses in angle resolution or signal sensitivity.

The membrane-based torque magnetometer allows for a broad range of applications and has the potential for multisensoric techniques. By applying a gradient field, it is also possible to investigate magnetically isotropic samples. Furthermore, the setup can be easily adjusted to allow torque-detected electron spin resonance and photoinduced measurements by replacing the piezoelectric chip with a piezo ring, as this makes the sample accessible by terahertz radiation and visible light. It is also possible to guide a second fiber towards the membrane for irradiation of the sample crystal. Additional potential lies within the flatness of the membrane surface which is suitable for thin film deposition and enables deposition of a small temperature sensor directly on the membrane. ${ }^{23}$ This even offers the possibility for differential nanocalorimetric measurements for investigations of phase transitions.

\section{ACKNOWLEDGMENTS}

The work was funded by the Deutsche Forschungsgemeinschaft (DFG) via No. DR228/43-1 in the frame of the Schwerpunktprogramm No. 1601 "New frontiers in sensitivity for EPR spectroscopy: from biological cells to nano materials." L.B. acknowledges support by the Alexander von Humboldt Foundation via the Sofja Kovalevskaja Award.

\section{APPENDIX A: ANALYTIC SOLUTION FOR MEMBRANE DEFLECTION}

The analytical solution presented by Eaton et al. ${ }^{18}$ provides a powerful tool to estimate the response of a clamped circular membrane for large deflections under the influence of built-in stresses and a uniformly distributed load. Even though the solution holds for circular membranes, it still provides a good approximation for a square membrane with comparable dimensions.

The fact that the analytical approach accounts for builtin stresses is particularly important in our case, since the SiN membranes are produced with a tensile stress of $\leq 250 \mathrm{MPa} .{ }^{25}$ This inner stress significantly influences the response of the membrane to a load. For small deflections of less than $1 / 5$ of the membrane thickness, the solution is well known and the deflection $w$ under a uniform pressure $P$ is given by ${ }^{39}$

$$
w_{\text {small }}(r)=\frac{P a^{4}}{64 D}\left[1-\left(\frac{r}{a}\right)^{2}\right]^{2},
$$

with $r$ the radial coordinate, $a$ the membrane radius, and the flexural rigidity $D$ given by

$$
D=\frac{E h^{3}}{12\left(1-v^{2}\right)},
$$

where $E$ is Young's modulus, $h$ is the plate thickness, and $v$ is Poisson's ratio.

From now on we will only discuss the deflection $w$ at the center of the plate $r=0$. In small deflection theory, $w_{\text {small }}$ is linear with respect to the applied pressure. This of course cannot be true for larger deflections where the relation is highly non-linear due to intrinsic stresses introduced by the deflection. By taking these stresses and a built-in residual strain $\varepsilon_{i}$ into account, Eaton et al. derived the following solution for the maximal deflection $w_{\text {large }}$ at the center of a circular membrane: ${ }^{18}$ 


$$
\begin{aligned}
w_{\text {large }} & =\sqrt[3]{-\frac{\beta}{2}+\gamma}+\sqrt[3]{-\frac{\beta}{2}-\gamma}, \\
\gamma & =\sqrt{\frac{\alpha^{3}}{27}+\frac{\beta^{2}}{4}}, \\
\alpha & =14 \frac{4 h^{2}+3 a^{2} \varepsilon_{i}(1+v)}{(1+v)(23-9 v)}, \\
\beta & =\frac{-7 P a^{4} h^{2}}{8 D(1+v)(23-9 v)} .
\end{aligned}
$$

The solution is found to be in good agreement with finite element analysis and experimental data of $\mathrm{aSi}_{3} \mathrm{~N}_{4}$ pressure sensor with a radius $a=50 \mu \mathrm{m}$ and a thickness $h=1.2 \mu \mathrm{m}$.

In order to relate this solution to a square membrane, we compared it with a solution for clamped rectangular plates derived by Timoshenko and Woinowsky-Krieger. ${ }^{39}$ This solution is based on the suggestion by Lévy ${ }^{40}$ that the solution $u$ of the equation of deflection,

$$
\frac{\partial^{4} u}{\partial x^{4}}+2 \frac{\partial^{4} u}{\partial x^{2} \partial y^{2}}+\frac{\partial^{4} u}{\partial y^{4}}=\frac{q}{D},
$$

with an arbitrary load $q$, takes the form of a series,

$$
u=\sum_{m=1}^{\infty} Y_{m} \sin \frac{m \pi x}{a},
$$

where $a$ is the edge length of a square plate in the $x$ - $y$-plane and $Y_{m}$ is a function of $y$ only. Timoshenko and WoinowskyKrieger obtain a dependence for the deflection at the center of the plate under uniform pressure $P$ of the form:

$$
u_{\text {small }}=\alpha \frac{P a^{4}}{D},
$$

where $\alpha$ is determined as $\alpha=0.00126$, which is confirmed by recent calculations. ${ }^{41}$ The solution takes the same form as the one for circular plates, except for a slightly different prefactor. The solution $u_{\text {small }}$ is only valid for small deflections. In order to estimate the response $u_{\text {large }}$ of a clamped square membrane for large deflections and a residual built-in strain $\varepsilon_{i}$, we make the assumption,

$$
\frac{u_{\text {large }}}{u_{\text {small }}}=\frac{w_{\text {large }}}{w_{\text {small }}} .
$$

The solution is shown in Fig. 8(a) for different values of $\varepsilon_{i}$. It is evident that the deflection behaves linearly with an applied load for deflections smaller $5 \mu \mathrm{m}$. Additionally, from Eq. (A3), we get that the deflection behaves inversely proportional to the thickness $h$ of the membrane. By using a thinner membrane with a thickness of $50 \mathrm{~nm}$, the sensitivity of the membrane-based torque magnetometer can be improved by a factor of 2 .

\section{APPENDIX B: SQUID MAGNETIZATION MEASUREMENTS}

The saturation magnetic moment of the single crystal with a mass of $m=(2.2 \pm 1.0) \mu \mathrm{g}$ was determined beforehand by an additional SQUID measurement to be $M \approx 1.3 \times 10^{-7} \mathrm{Am}^{2}$; in excellent agreement with the value expected using the molar mass of $\mu_{M}=2060 \mathrm{~g} / \mathrm{mol}$. As seen from Fig. 9, quantum
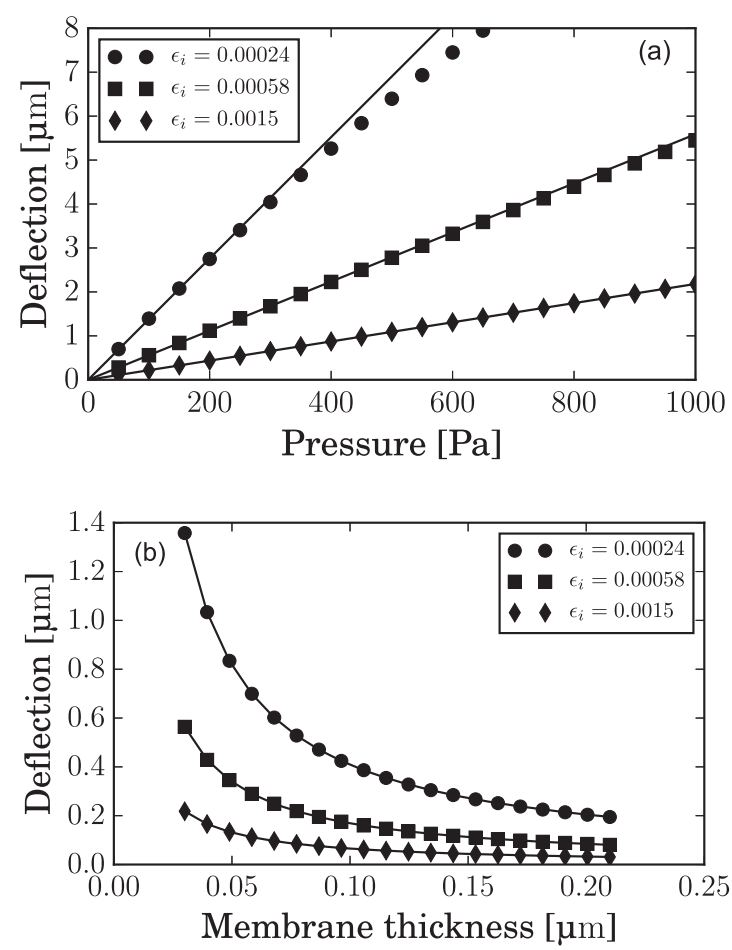

FIG. 8. (a) The deflection of the membrane reveals a linear behavior under an applied load for deflections smaller than $5 \mu \mathrm{m}$. The solid lines, corresponding to a linear fit, serve as a guide to the eye. The tensile stress related to a residual built-in strain $\varepsilon_{i}$ strongly affects the response of the membrane. (b) The deflection behaves inversely proportional to the thickness $h$ of the membrane. The solid line corresponds to a $1 / h$ fit to $u_{\text {large }}$,

tunneling steps can barely be resolved by the SQUID magnetometer, mainly due to the slow response time; in contrast to the measurements with our novel membrane-based torque magnetometer (see Figs. 7 and 10).

\section{APPENDIX C: SENSITIVITY}

For estimating the sensitivity of the membrane-based torque magnetometer, we used a crystalline single molecule magnet $\mathrm{Fe}_{3} \mathrm{Cr}^{42}$ From SQUID measurement the magnetic moment was determined to $M=1.7 \times 10^{-7} \mathrm{Am}^{2}$. In a magnetic field of $0.12 \mathrm{~T}$ and at a temperature of $1.9 \mathrm{~K}$ the maximal deflection of the membrane at an angle of $45^{\circ}$ of the easy axis to the field axis was $130 \mathrm{~nm}$. The noise level of the output voltage was $10 \mathrm{mV}$, corresponding to a deflection noise of $0.25 \mathrm{~nm}$, leading to a signal-to-noise ratio (SNR) of 520. Since the magnetic torque behaves linearly with magnetic field up to approximately $1.2 \mathrm{~T}$, we would increase SNR to 5200 at this field. Furthermore, by using a membrane with a thickness of $50 \mathrm{~nm}$, the sensitivity gets improved by a factor of 2 (see Fig. 8). This gives a SNR of 10400 for a magnetic moment of $M=1.7 \times 10^{-7} \mathrm{Am}^{2}$ resulting in a detectable magnetic moment smaller than $1.6 \times 10^{-11} \mathrm{Am}^{2}$. The bandwidth of the measurement is limited by the integration time of 0.2 ms of the Keithley 2400, corresponding to a bandwidth of $\Delta f=5 \mathrm{kHz}$ (The bandwidth of the photodiode is $\Delta f=50$ $\mathrm{kHz}$ ). This results in a sensitivity of $2.3 \times 10^{-13} \mathrm{Am}^{2} / \sqrt{\mathrm{Hz}}=$ $2.5 \times 10^{10} \mu_{B} / \sqrt{\mathrm{Hz}}$ at $1.2 \mathrm{~T}$. For comparison, the commercial torque magnetometer option P550 for a PPMS (Physical 

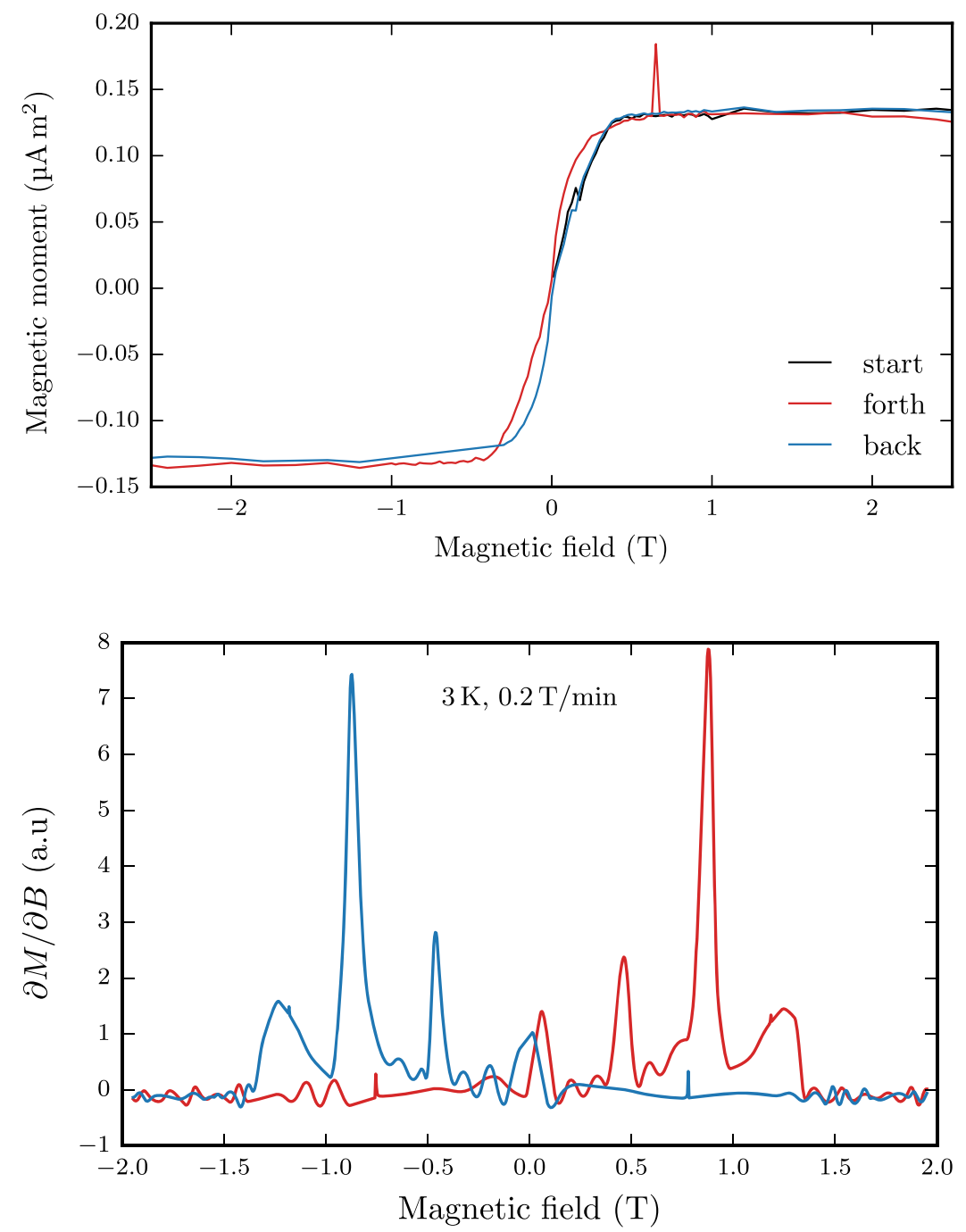

FIG. 9. Field-dependent magnetization measurements of the $\mathrm{Mn}_{12}$ ac single crystal at $T=3 \mathrm{~K}$ performed by a conventional SQUID magnetometer MPMS-XL7, QuantumDesign. A small hysteresis gap can be identified; however, quantum-tunneling steps remain basically unresolved, mainly due to the slow response time.
FIG. 10. The derivative $\mathrm{d} M / \mathrm{d} B$ as a function of applied magnetic fields is a common method to identify steps in the magnetization curve. Exemplarily, the derivative of the measurement shown in Fig. 7 at $3 \mathrm{~K}$ with a sweep rate of $0.2 \mathrm{~T} / \mathrm{min}$ is displayed.
Properties Measurement System, Quantum Design) offers a sensitivity of about $5 \times 10^{-9} \mathrm{Am}^{2} / \sqrt{\mathrm{Hz}}$ at $1.2 \mathrm{~T}$. Whereas the MPMS 3 SQUID magnetometer (QuantumDesign) provides a sensitivity of about $1 \times 10^{-11} \mathrm{Am}^{2} / \sqrt{\mathrm{Hz}}$.

Further, we would like to note that the noise level of $10 \mathrm{mV}$ is mainly caused by turbulent helium flow. By encapsulating the membrane sensor, we expect an improvement of noise level of about a factor of 4 .

${ }^{1}$ P. Ripka, Magnetic Sensors and Magnetometers (Artech House, Boston, Massachusetts, 2000).

${ }^{2}$ S. Tumanski, Handbook of Magnetic Measurements (CRC Press, Boca Raton, FL, 2011).

${ }^{3}$ A. Grosz, M. J. Haji-Sheikh, and S. Mukhopadhyay, High Sensitivity Magnetometers: Smart Sensors, Measurement and Instrumentation (Springer, Berlin, 2016).

${ }^{4}$ M. J. Naughton, J. P. Ulmet, A. Narjis, S. Askenazy, M. V. Chaparala, and A. P. Hope, "Cantilever magnetometry in pulsed magnetic fields," Rev. Sci. Instrum. 68, 4061 (1997).

${ }^{5}$ C. Rossel, M. Willemin, A. Gasser, H. Bothuizen, G. I. Meijer, and H. Keller, "Torsion cantilever as magnetic torque sensor," Rev. Sci. Instrum. 69, 3199-3203 (1998).

${ }^{6}$ M. Willemin, C. Rossel, J. Brugger, M. H. Despont, H. Rothuizen, P. Vettiger, J. Hofer, and H. Keller, "Piezoresistive cantilever designed for torque magnetometry," J. Appl. Phys. 83, 1163 (1998).

${ }^{7}$ J. Rigue, D. Chrischon, A. de Andrade, and M. Carara, "A torque magnetometer for thin films applications," J. Magn. Magn. Mater. 324, 1561-1564 (2012).
${ }^{8}$ M. R. Schaapman, P. C. M. Christianen, J. C. Maan, D. Reuter, and A. D. Wieck, "A multipurpose torsional magnetometer with optical detection," Appl. Phys. Lett. 81, 1041-1043 (2002).

${ }^{9}$ F. El Hallak, J. van Slageren, and M. Dressel, "Torque detected broad band electron spin resonance," Rev. Sci. Instrum. 81, 095105 (2010).

${ }^{10}$ F. El Hallak, P. Neugebauer, A. L. Barra, J. Van Slageren, M. Dressel, and A. Cornia, "Torque-detected ESR of a tetrairon(III) single molecule magnet," J. Magn. Reson. 223, 55-60 (2012).

${ }^{11}$ J. P. Davis, D. Vick, D. C. Fortin, J. A. J. Burgess, W. K. Hiebert, and M. R. Freeman, "Nanotorsional resonator torque magnetometry," Appl. Phys. Lett. 96, 072513 (2010).

${ }^{12}$ T. Firdous, D. Vick, M. Belov, F. F. Sani, A. McDermott, J. Losby, D. Bazylinski, T. Prozorov, D. Potter, and M. Freeman, "Nanomechanical torque magnetometry of an individual aggregate of 350 nanoparticles," Can. J. Phys. 93, 1252-1256 (2015).

${ }^{13}$ M. Dörfel, M. Kern, H. Bamberger, P. Neugebauer, K. Bader, R. Marx, A. Cornia, T. Mitra, A. Müller, M. Dressel, L. Bogani, and J. van Slageren, "Torque-detected electron spin resonance as a tool to investigate magnetic anisotropy in molecular nanomagnets," Magnetochemistry 2, 25 (2016).

${ }^{14}$ J. Brugger, M. Despont, C. Rossel, H. Rothuizen, P. Vettiger, and M. Willemin, "Microfabricated ultrasensitive piezoresistive cantilevers for torque magnetometry," Sens. Actuators, A 73, 235-242 (1999).

${ }^{15} \mathrm{E}$. Ohmichi and T. Osada, "Torque magnetometry in pulsed magnetic fields with use of a commercial microcantilever," Rev. Sci. Instrum. 73, 3022-3026 (2002).

${ }^{16} \mathrm{H}$. Takahashi, K. Ishimura, T. Okamoto, E. Ohmichi, and H. Ohta, "New method for torque magnetometry using a commercially available membrane-type surface stress sensor," J. Phys. Soc. Jpn. Part 2 86, 063002 (2017). 
${ }^{17}$ A. Cornia, D. Gatteschi, and R. Sessoli, "New experimental techniques for magnetic anisotropy in molecular materials," Coord. Chem. Rev. 219-221, 573-604 (2001).

${ }^{18}$ W. P. Eaton, F. Bitsie, J. Smith, and D. Plummer, "A new analytical solution for diaphragm deflection and its application to a surace-micromachined pressure sensor," presented at International Conference on Modeling and Simulation of Microsystems MSM99' (Computational Publications, 1999), pp. 640-643.

${ }^{19}$ A. D. Drake and D. C. Leiner, "Fiber-optic interferometer for remote subangstrom vibration measurement," Rev. Sci. Instrum. 55, 162-165 (1984).

${ }^{20}$ D. Rugar, H. J. Mamin, R. Erlandsson, J. E. Stern, and B. D. Terris, "Force microscope using a fiber-optic displacement sensor,” Rev. Sci. Instrum. 59, 2337-2340 (1988).

${ }^{21}$ D. Rugar, H. J. Mamin, and P. Guethner, "Improved fiber-optic interferometer for atomic force microscopy," Appl. Phys. Lett. 55, 2588-2590 (1989).

${ }^{22}$ B. M. Zwickl, W. E. Shanks, A. M. Jayich, C. Yang, A. C. B. Jayich, J. D. Thompson, and J. G. E. Harris, "High quality mechanical and optical properties of commercial silicon nitride membranes," Appl. Phys. Lett. 92, 103125 (2008).

${ }^{23}$ S. Tagliati, "Membrane-based nanocalorimetry for low temperature studies with high resolution and absolute accuracy," Doctoral thesis, Stockholm University, 2011.

${ }^{24}$ N. Scozzaro, W. Ruchotzke, A. Belding, J. Cardellino, E. Blomberg, B. McCullian, V. Bhallamudi, D. Pelekhov, and P. Hammel, "Magnetic resonance force detection using a membrane resonator," J. Magn. Reson. 271, 15-20 (2016)

${ }^{25}$ Norcada, Inc., Canada, Silicin Nitride Windows.

${ }^{26}$ A. Cornia, M. Affronte, A. G. M. Jansen, D. Gatteschi, A. Caneschi, and R. Sessoli, "Magnetic anisotropy of $\mathrm{Mn}_{12}$-acetate nanomagnets from high-field torque magnetometry," Chem. Phys. Lett. 322, 477-482 (2000).

${ }^{27}$ QPhotonics LLC, USA, DFB laser diode QDFBLD-1550-5.

${ }^{28}$ Thorlabs GMBH, Germany.

${ }^{29}$ FEMTO Messtechnik GmbH, Germany, Photoreceiver OE-200-IN2.

${ }^{30}$ G. Martinet, S. Blivet, F. Chatelet, M. Fouaidy, N. Hammoudi, A. Olivier, and H. Saugnac, "Low temperature properties of piezoelectric actuators used in SRF cavities cold tuning systems," in Proceeding EPAC (European Physical Society Accelerator Group, Edinburgh, 2006), pp. 390-392.

${ }^{31}$ D. Catteschi, R. Sessoli, and J. Villain, Molecular Nanomagnets (Oxford University Press, 2006).

${ }^{32}$ A. L. Barra, D. Gatteschi, and R. Sessoli, "High frequency EPR spectra of a molecular nanomagnet provide a key to understand quantum tunneling of the magnetization," Phys. Rev. B 56, 8192-8198 (1997).

${ }^{33}$ A. Caneschi, D. Gatteschi, and R. Sessoli, "Alternating current susceptibility, high field magnetization, and millimeter band EPR evidence for a ground $S=10$ state in $\left[\mathrm{Mn}_{12} \mathrm{O}_{12}\left(\mathrm{CH}_{3} \mathrm{COO}\right)_{16}\left(\mathrm{H}_{2} \mathrm{O}\right)_{4}\right] \cdot 2 \mathrm{CH}_{3} \mathrm{COOH} \cdot 4 \mathrm{H}_{2} \mathrm{O}$," J. Am. Chem. Soc. 113, 5873-5874 (1991).

${ }^{34}$ M. Dressel, B. Gorshunov, K. Rajagopal, S. Vongtragool, and A. A. Mukhin, "Quantum tunneling and relaxation in $\mathrm{Mn}_{12}$-acetate studied by magnetic spectroscopy," Phys. Rev. B 67, 060405 (2003).

35 J. van Slageren, S. Vongtragool, B. Gorshunov, A. Mukhin, and M. Dressel, "Frequency-domain magnetic-resonance spectroscopic investigations of the magnetization dynamics in $\mathrm{Mn}_{12}$ Ac single crystals," Phys. Rev. B 79, 224406 (2009).

${ }^{36}$ The data taken at $3 \mathrm{~K}$ rather resemble data taken at lower temperatures of about $2.5 \mathrm{~K}$. We cannot rule out a small offset in temperature between the sample and sensor or a small error in the sensor calibration.

${ }^{37}$ J. R. Friedman, M. P. Sarachik, and R. Ziolo, "Macroscopic measurement of resonant magnetization tunneling in high-spin molecules," Phys. Rev. Lett. 76, 3830-3833 (1996).

${ }^{38}$ L. Thomas, F. Lionti, R. Ballou, D. Gatteschi, R. Sessoli, and B. Barbara, "Macroscopic quantum tunnelling of magnetization in a single crystal of nanomagnets," Nature 383, 145-147 (1996).

${ }^{39}$ S. Timoshenko and S. Woinowsky-Krieger, The Theory of Plates and Shells, Classic Textbook Reissue Series, 2nd ed. (McGraw-Hill, 1964).

${ }^{40}$ M. Lévy, "Sur l'équilibre élastique d'une plaque rectangulaire," Comptes Rendus l'Acad. Sci. Paris 129, 535-539 (1899).

${ }^{41}$ C. E. İmrak and I. Gerdemeli, "An exact solution for the deflection of a clamped rectangular plate under uniform load," Appl. Math. Sci. 1, 2129-2137 (2007).

${ }^{42}$ E. Tancini, M. J. Rodriguez-Douton, L. Sorace, A.-L. Barra, R. Sessoli, and A. Cornia, "Slow magnetic relaxation from hard-axis metal ions in tetranuclear single-molecule magnets," Chem. - Eur. J. 16, 10482-10493 (2010). 